ISSN 0103-5150

Fisioter. Mov., Curitiba, v. 24, n. 3, p. 463-470, jul./set. 2011 Licenciado sob uma Licença Creative Commons

\title{
Análise do pico de ativação do glúteo máximo na marcha em mulheres com instabilidade do tornozelo
}

\author{
Analysis of the maximum gluteus activation during \\ women walk with ankle instability
}

\author{
Lygia Paccini Lustosa ${ }^{[a]}$, Ana Paula Miranda Furbino ${ }^{[b]}$, Camila Santos Cruz ${ }^{[b]}$, \\ Ian Lara Lamounier Andrade ${ }^{[c]}$, Cláudia Venturini ${ }^{[\mathrm{d}]}$
}

[a] Doutora em Ciências da Reabilitação, professora do curso de Fisioterapia do Centro Universitário de Belo Horizonte (UNI-BH), Centro Universitário Newton Paiva, Belo Horizonte, MG - Brasil, e-mail: lpaccini@horizontes.net

[b] Bacharel em Fisioterapia, Centro Universitário de Belo Horizonte (UNI-BH), Belo Horizonte, MG - Brasil.

[c] Mestre em Ciências da Reabilitação, professor do curso de Fisioterapia do Centro Universitário de Belo Horizonte (UNI-BH), Belo Horizonte, MG - Brasil.

[d] Mestre em Ciências da Reabilitação, professora do curso de Fisioterapia da Pontifícia Universidade Católica de Minas Gerais (PUC Minas), Betim, MG - Brasil.

\section{Resumo}

Introdução: 0 risco de recidiva após a entorse de tornozelo pode estar associado com modificações da estabilidade postural e do recrutamento muscular das articulações do quadril e do tornozelo. Objetivos: Avaliar o pico de ativação muscular do glúteo máximo durante a marcha em esteira, em mulheres, comparando voluntárias com história de entorse grau II de tornozelo, com um grupo de voluntárias sem história de entorse. Materiais e métodos: Participaram 26 mulheres, jovens, sendo 13 voluntárias com história de entorse unilateral de tornozelo e 13 sem história de entorse. A ativação do músculo glúteo máximo foi avaliada por meio do eletromiógrafo de superfície EMG System do Brasil durante a marcha em esteira. Resultados: No grupo com história de entorse não houve diferença significativa na medida percentual de ativação normalizada do glúteo máximo durante a marcha, quando comparou-se membro acometido e não acometido $(p>0,57)$. No grupo sem história de entorse houve diferença significativa entre os membros direito e esquerdo na medida percentual de ativação normalizada do glúteo máximo durante a marcha ( $\mathrm{p}=$ $0,01)$. Quando comparados os grupos, não houve diferença significativa entre membro acometido e membros direito e esquerdo do grupo sem história de entorse $(p>0,51)$. Conclusão: Pela ausência de diferença 
entre os grupos pode-se supor que existam fatores adaptativos, como musculares, neuromusculares e dominância dos membros, que determinam uma adaptação após a entorse do tornozelo, possibilitando uma atividade da marcha adequada.

Palavras-chave: Instabilidade articular. Tornozelo. Eletromiografia. Mulheres.

\begin{abstract}
Introduction: The return risk, after ankle sprain, can be associated with modifications of the postural stability and the muscular strength of the hip and the ankle. Objectives: The aim of this study was to evaluate the maximum gluteus activation during the walk on the treadmill, in women, comparing volunteers with second degree of ankle sprain history, with a group of volunteers with no sprain history. Materials and methods: Twenty-six young women were selected for the study, being 13 individuals with unilateral ankle sprain history and another 13 with no sprain history. The maximum gluteus activation was evaluated by the electromyography of surface $(E M G)$, during the walk on the treadmill. Results: On the group with sprain history there was not any significant difference on the measure of normal activation of the maximum gluteus during the walk, when compared unstable member and no unstable member $(p>0,57)$. However, on the group with no sprain history there was a significant difference between left and right members on the measure of normal activation of the maximum gluteus during the walk $(p=0,01)$. When comparing both groups, there was not any significant difference between unstable member and the others two members of the no sprain history group ( $p>051)$. Conclusion: The results can be suggested that exist muscular, neuromuscular and sideways factors that can be determinate an adaptation after the ankle sprain, enabling a convenient walk activity.
\end{abstract}

Keywords: Joint instability. Ankle. Electromyography. Women.

\section{Introdução}

A entorse de tornozelo é uma lesão frequente no meio esportivo e nas atividades de vida diária e, geralmente, está entre as lesões traumáticas mais prevalentes (1-5). A entorse em inversão é a mais comum dentre os mecanismos de lesão, graças à somatória de fatores relacionados com as estruturas ósseas, musculares e ligamentares (1, 3, 6-8). Mangwani et al. estabeleceram que o ligamento talofibular anterior rompe-se isoladamente em $66 \%$ dos casos, enquanto o ligamento calcâneo fibular é comprometido em $20 \%$ dos casos (9). Sabe-se ainda que aproximadamente $80 \%$ dos indivíduos apresentam lesão recorrente do tornozelo após o primeiro episódio do entorse $(5,10)$, e que a entorse responsável pela instabilidade acontece quando ocorre a lesão combinada do ligamento talofibular anterior e o calcâneo fibular $(5,9)$.

A instabilidade crônica do tornozelo é desencadeada após a lesão ligamentar e é difícil de ser avaliada e a falta de um teste considerado padrão ouro dificulta o diagnóstico clínico (10). Konradsen afirmou que alguns indivíduos podem apresentar alterações proprioceptivas do tornozelo, o que influenciaria e facilitaria novas recidivas, com o mesmo mecanismo de lesão (11). Dentro desse contexto, alguns autores demonstraram a existência de fatores predisponentes, como o déficit de força dos músculos fibulares e/ ou dos abdutores do quadril, a frouxidão ligamentar $(5,7,12)$ e a posteriorização da fíbula (7). Dessa forma, a investigação da instabilidade crônica da articulação do tornozelo tornou-se foco de atenção na literatura, no intuito de esclarecer os fatores causais e as suas alterações secundárias $(13,14)$, investigando ainda as consequências em outras articulações (15).

Existem evidências demonstrando que indivíduos tratados evoluem para a estabilidade da articulação após uma lesão ligamentar parcial (9). Da mesma forma, indivíduos não tratados podem desenvolver compensações e alterações posturais como forma de adaptação à disfunção na busca da funcionalidade (13). Nesse contexto, existem inferências sobre modificações da estabilidade postural e do recrutamento muscular das articulações do quadril e do tornozelo, após a entorse de tornozelo, que podem aumentar o risco de recidiva (5). Sendo assim, alguns autores apontaram para a importância da análise da marcha 
em indivíduos que apresentam instabilidade, na tentativa de esclarecer a influência das cadeias musculares que ligam o tornozelo à coluna lombar e que poderiam constituir vias de compensações musculares (13). Dessa forma, compreender a variedade de circunstâncias comuns que podem afetar o quadril e o tornozelo, estabelecendo os papéis funcionais dos músculos que agem nessas articulações durante a marcha, pode ajudar a esclarecer fatores causais e estabelecer melhores intervenções clínicas. Esse entendimento pode ainda contribuir para explicar a interação entre os músculos do quadril e do tornozelo na análise dos mecanismos compensatórios durante a marcha (8).

Diante disso, o objetivo deste estudo foi avaliar o pico de ativação do músculo glúteo máximo durante a marcha em esteira, em mulheres, comparando voluntárias com história de entorse do tornozelo em inversão, grau II, com um grupo de voluntárias sem história de entorse.

\section{Materiais e métodos}

Trata-se de um estudo de corte transversal com uma amostra de conveniência. Todas as voluntárias assinaram o Termo de Consentimento Livre e Esclarecido, aprovado pelo Comitê de Ética em Pesquisa do Centro Universitário de Belo Horizonte UNI-BH, sob o Parecer número 064/2006. Elas foram recrutadas por meio de cartazes afixados em uma escola de ensino superior particular de Belo Horizonte.

\section{Amostra}

Foram avaliadas 26 mulheres, divididas em dois grupos, sendo 13 voluntárias com história de entorse do tornozelo em inversão, grau II, unilateral e 13 sem história de entorse.

0 grupo com história de entorse de tornozelo teve como critério de inclusão: idade entre 20 a 30 anos; história de entorse do tornozelo unilateral há mais de três meses, classificada em grau II; não ter realizado tratamento fisioterápico pós-entorse; não ter queixa de dor lombar nos últimos três meses; ser sedentária ou praticar atividade física há menos de três meses por menos de três vezes na semana. Considerou-se entorse grau II aquelas que relataram ter tido apenas um episódio de entorse com consequente perda funcional após o episódio e, ainda, edema distal, região de equimose e limitação de movimentos, com melhora de todos esses sinais e sintomas em até três semanas.

O grupo sem história de entorse de tornozelo teve os mesmos critérios de inclusão que o grupo com entorse, exceto ter história de entorse do tornozelo.

Os critérios de exclusão para ambos os grupos foram: indivíduos submetidos a qualquer tipo de cirurgia ortopédica e/ou que fraturaram membros inferiores; informar a presença de doenças arteriais e/ou venosas periféricas com diagnóstico prévio; $\mathrm{IMC} \geq 30$; relatar história de entorse com sinais e sintomas diferentes dos considerados para inclusão; relatar mais de um episódio de entorse; relatar processo alérgico, como dermatite de contato e/ou alergia a esparadrapo, álcool e/ou gel condutor.

\section{Instrumentos}

Utilizou-se um dinamômetro Instrutherm DD100 (margem de erro de $0,4 \%$ segundo o fabricante), confiável e válido para a medida da força muscular isométrica durante a contração voluntária máxima (CVM), associado ao registro eletromiográfico.

0 pico de ativação muscular do glúteo máximo durante a marcha foi registrado por meio de um eletromiógrafo de superfície de oito canais EMG System do Brasil, com o sinal filtrado por passa banda de 20$500 \mathrm{~Hz}$, amplificado em 1.000 vezes e com relação de rejeição do modo comum > $120 \mathrm{~dB}$. Utilizaram-se eletrodos bipolares ativos com distância de $02 \mathrm{~cm}$ entre eles. Os dados foram processados usando o software específico do próprio eletromiógrafo. A atividade elétrica foi mensurada pelo pico de ativação do glúteo máximo usando os valores da root mean square (RMS).

A marcha foi realizada em uma esteira elétrica Moviment Tecnology com dimensões $100 \times 160 \mathrm{~cm}$, em velocidade de $3,6 \mathrm{~km} / \mathrm{h}$, na tentativa de reproduzir uma marcha confortável. Essa velocidade foi considerada confortável pelo fato de ser uma velocidade média possível para a população na idade estudada, sem necessidade de sobrecarga, ou seja, uma marcha desenvolvida no dia a dia, para execução de tarefas simples.

\section{Procedimentos}

As voluntárias foram avaliadas quanto à frouxidão articular do tornozelo por um único avaliador. 
Esse examinador é fisioterapeuta, com mais de 18 anos de experiência no meio clínico, na reabilitação ortopédica e em terapia manual. $\mathrm{O}$ avaliador não teve conhecimento prévio da presença ou ausência de história de entorse de tornozelo nas voluntárias, permanecendo como avaliador mascarado. Dessa forma, ele realizou os testes ortopédicos do tilt talar (16) e da gaveta anterior (16) e informou, segundo a percepção da mobilidade acessória, o grau de frouxidão articular. Testes de confiabilidade teste/ reteste já tinham sido realizados anteriormente, por esse profissional, durante o desenvolvimento de sua dissertação de mestrado $(17,18)$.

Em seguida, a voluntária foi convidada a deitarse em uma maca em decúbito ventral, onde foi realizada a palpação da maior região de ventre muscular do glúteo máximo para a colocação dos eletrodos. A colocação simétrica bilateralmente dos eletrodos foi garantida com a utilização de uma fita métrica padrão, para a medida da distância média da espinha ilíaca póstero-superior até o trocânter maior do fêmur ipsilateral $(19,20)$. Esse procedimento foi realizado por apenas um avaliador, treinado previamente. Antes da colocação dos eletrodos foi realizada limpeza com álcool, antes e após a tricotomia do local, para minimizar a influência da impedância pele/eletrodo $(19,20)$. Foram colocados dois eletrodos de cada lado na massa glútea, com uma distância de $2 \mathrm{~cm}$ de um centro ao outro. Utilizou-se a referência anatômica como recomendado pela Surface Electromyography for the Non-Invasive Assessment of Muscle (Seniam) (19). Um eletrodo de referência foi posicionado na espinha ilíaca ântero-superior à direita.

A medida da CVM foi realizada estando a voluntária em decúbito ventral, com o dinamômetro posicionado perpendicular à maca, preso a um velcro de $5 \mathrm{~cm}$ de largura, posicionado $3 \mathrm{~cm}$ superior à fossa poplítea. Outro velcro, também de $5 \mathrm{~cm}$ de largura, foi posicionado na região escapular para impedir a compensação por meio da extensão de tronco. A voluntária foi solicitada a fazer flexão de joelho em $90^{\circ}$ e posicionar as mãos na região occipital, para então realizar a extensão de quadril (Figura 1). 0 dinamômetro foi zerado. Sempre o mesmo pesquisador proporcionou o mesmo feedback, por meio da visualização do visor do aparelho e estímulo verbal durante o movimento de extensão de quadril durante uma contração voluntária máxima isométrica. 0 procedimento foi realizado por três vezes com intervalos de

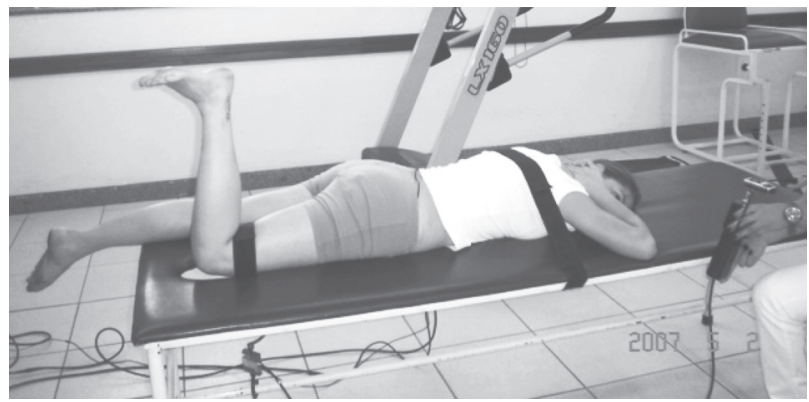

Figura 1 - Contração voluntária máxima, com feedback visual

um minuto entre cada uma das medidas. 0 sinal eletromiográfico foi registrado durante as três contrações e a média do maior registro foi utilizada para a normalização dos dados.

Na sequência, foi solicitado às voluntárias que se posicionassem na esteira elétrica para realizar a marcha. Elas permaneceram com o tênis de uso diário e iniciaram a caminhada até alcançar a velocidade de $3,6 \mathrm{~km} / \mathrm{h}$ no primeiro minuto de caminhada. Essa velocidade foi mantida durante um período de três minutos e depois foi realizada a desaceleração até a parada total da esteira. 0 registro eletromiográfico foi feito a partir do segundo minuto da marcha por um período de 30 segundos. Um examinador permaneceu sempre ao lado da voluntária. Em nenhum momento a voluntária foi informada sobre quando iria iniciar o registro eletromiográfico e o disparo do aparelho foi feito por um examinador independente, que permaneceu no computador, controlando o tempo da marcha e o eletromiógrafo. Não foi utilizado nenhum tipo de registro para as fases da marcha.

\section{Redução dos dados}

0 nível de frouxidão articular foi relatado pelo avaliador mascarado por percepção do movimento acessório de deslizamento em milímetros, conforme sua experiência clínica, sendo considerados: valores menores ou iguais a cinco milímetros - estável; valores entre seis a dez milímetros - instabilidade moderada; e valores maiores que dez milímetros instabilidade grave (16).

A frequência de amostragem do sinal foi realizada em $1.000 \mathrm{~Hz}$, utilizando-se filtros passa banda de 20 a $500 \mathrm{~Hz}$, uma vez que $80 \%$ da densidade espectral de potência encontra-se nessa amplitude de frequência $(20,21)$. 
Todos os dados foram processados em um software específico de processamento de sinais do próprio eletromiógrafo. Para os registros eletromiográficos, foi utilizada a CVM como referência para normalização. Utilizou-se uma janela de tempo sobreposta, de um décimo de segundo (100 ms), sobre a área gráfica de melhor ativação muscular selecionada visualmente para análise da root mean square (RMS). Os maiores valores da RMS na CVM foram selecionados, assim como os maiores valores dos registros da marcha. Dessa forma, optou-se pela análise do pico de atividade elétrica durante a marcha na esteira, registrado dentro de um intervalo de 30 segundos, após um minuto do início a caminhada.

\section{Análise estatística}

A idade das voluntárias e o IMC foram analisados de forma descritiva, com cálculo de média e desviopadrão. A comparação entre grupos, com e sem história de entorse, do maior pico de ativação durante a marcha normalizada pela CVM foi realizada por meio do teste $t$ de Student, para amostras independentes, após confirmação da normalidade dos dados por meio do teste de Shapiro-Wilk. A comparação entre membro acometido e não acometido e membro direito e esquerdo foi realizada por meio do teste t de Student pareado. 0 nível de significância foi considerado $\alpha=0,05$.

\section{Resultados}

O grupo com história de entorse do tornozelo ( $\mathrm{n}=13$ ) apresentou média de idade de 23,7 anos $( \pm 2,9)$ e média do IMC de $22,6 \mathrm{~kg} / \mathrm{m}^{2}( \pm 2,9)$. 0 tempo de entorse relatado pelas voluntárias apresentou média de 27,6 meses $( \pm 4,9)$. Nove $(69,2 \%)$ voluntárias apresentaram entorse em inversão à direita; e quatro $(30,8 \%)$ à esquerda. 0 grupo sem história de entorse $(\mathrm{n}=13)$ apresentou média de idade de 23,5 anos $( \pm 2,6)$ e média de IMC de $20,8 \mathrm{~kg} / \mathrm{m}^{2}( \pm 2,5)$. As características dos dois grupos encontram-se na Tabela 1.

No grupo com história de entorse, quanto à comparação eletromiográfica entre membro acometido e não acometido, não houve diferença significativa na medida percentual de ativação do glúteo máximo durante a marcha normalizada pela CVM $(\mathrm{p}=0,57)$ (Tabela 2).
No grupo sem história de entorse, houve diferença significativa entre os membros direito e esquerdo na medida percentual de ativação do glúteo máximo durante a marcha normalizada pela CVM $(\mathrm{p}=$ 0,01) (Tabela 2).

Tabela 1 - Dados relativos à caracterização do grupo com entorse e do grupo sem entorse do tornozelo

\begin{tabular}{lcc}
\hline \multicolumn{3}{c}{ Caracterização da amostra } \\
\hline & $\begin{array}{c}\text { Grupo com } \\
\text { entorse }\end{array}$ & $\begin{array}{c}\text { Grupo sem } \\
\text { entorse }\end{array}$ \\
\hline Média idade (anos) & $23,7( \pm 2,9)$ & $23,5( \pm 2,5)$ \\
IMC (kg/m²) & $22,6( \pm 2,9)$ & $20,8( \pm 2,5)$ \\
Entorse tornozelo direito & $69,2 \%$ & - \\
Entorse tornozelo esquerdo & $30,8 \%$ & - \\
\hline
\end{tabular}

Fonte: Dados da pesquisa.

Tabela 2 - Média, desvio-padrão e valor estatístico comparando-se o pico de ativação do glúteo máximo durante a marcha em esteira, entre os membros, nos grupos com e sem entorse de tornozelo

\begin{tabular}{ccc}
\hline & Com entorse \\
\hline Acometido & Não acometido & $\mathbf{p}$ \\
\hline $18,8( \pm 11,1)$ & $17,4( \pm 9,8)$ & 0,57 \\
\hline \multicolumn{3}{c}{ Sem entorse } \\
\hline Direito & Esquerdo & $\mathbf{p}$ \\
\hline $18,9( \pm 5,1)$ & $15,3( \pm 5,6)$ & $0,01^{*}$ \\
\hline
\end{tabular}

Legenda: * = significativo $<0,05$.

Fonte: Dados da pesquisa.

Quando comparados os grupos, não houve diferença significativa entre o membro acometido do grupo com entorse com os membros direito e esquerdo do grupo sem história de entorse $(p>0,51)$. Também não houve diferença quando comparado o membro não acometido do grupo com entorse com os membros direito e esquerdo do grupo sem história de entorse $(p>0,97)$, demonstrando não haver diferença significativa, quanto à ativação do glúteo (Tabelas 3 e 4), entre os grupos. 
Tabela 3 - Média, desvio-padrão e valor de significância da comparação entre grupos com e sem entorse de tornozelo, considerando membro acometido comparado ao membro direito e esquerdo do grupo sem entorse

\begin{tabular}{ccccc}
\hline Com entorse & & \multicolumn{3}{c}{ Sem entorse } \\
\cline { 1 - 2 } \cline { 3 - 5 } Acometido & & Direito & Esquerdo & $\mathbf{p}$ \\
\hline $18,8( \pm 11,1)$ & & $18,9( \pm 5,1)$ & $15,3( \pm 5,6)$ & $>0,51$ \\
\hline
\end{tabular}

Fonte: Dados da pesquisa.

Tabela 4 - Média, desvio-padrão e valor de significância da comparação entre grupos com e sem entorse de tornozelo, considerando membro não acometido comparado ao membro direito e esquerdo do grupo sem entorse

\begin{tabular}{ccccc}
\hline Com entorse & \multicolumn{4}{c}{ Sem entorse } \\
\cline { 1 - 3 } \cline { 3 - 4 } Não acometido & Direito & Esquerdo & $\mathbf{p}$ \\
\hline $17,40( \pm 9,80)$ & & $18,90( \pm 5,10)$ & $15,30( \pm 5,60)$ & $>0,97$ \\
\hline
\end{tabular}

Fonte: Dados da pesquisa.

\section{Discussão}

Este estudo teve como objetivo comparar o pico de ativação dos glúteos durante a marcha, comparando mulheres jovens com e sem história de entorse do tornozelo. Os resultados demonstraram que não houve diferença de ativação entre membro acometido e não acometido durante a marcha, no grupo com história de entorse, assim como não houve diferença no pico de ativação do glúteo máximo, durante a marcha, quando comparados os grupos com e sem entorse.

Estudos biomecânicos demonstraram que durante a marcha é necessário ter força muscular, estabilidade de quadril e um bom posicionamento dos pés $(5,22)$. A colocação inadequada dos pés para realizar o apoio pode ser corrigida pela articulação subtalar, por meio da pronação e supinação, mas também pela ação do quadril, que trabalha em sinergismo com todo o membro inferior (22). Nesse contexto, para garantir uma boa estratégia postural utiliza-se a percepção inconsciente de posicionamento, que primeiramente é percebido pelo tornozelo, e depois por joelho, tronco e quadril, sucessivamente (23). No entanto, se existem alterações quanto à percepção desses movimentos e/ ou atraso de ativação ou resposta muscular, pode ocorrer uma estratégia alternativa (24), desencadeando, por exemplo, modificações cinemáticas. Esse pressuposto pode explicar em parte a ausência de diferença encontrada neste estudo, que sugere uma adaptação de estratégia de apoio no grupo com história de entorse de tornozelo.

Bullock-Saxton demonstrou que indivíduos com história de entorse do tornozelo apresentaram maior tempo para ativação do glúteo máximo do que indivíduos sem a história de instabilidade (25). 0 presente estudo não avaliou o tempo de ativação muscular ou outros parâmetros eletromiográficos, tendo como objetivo, pelo momento, apenas a avaliação do pico de ativação do glúteo máximo. A ausência de diferença entre os grupos e entre membros acometido e não acometido sugere que pode não existir alterações da estratégia de ativação muscular do glúteo máximo, durante a marcha, na população avaliada, mas não exclui a possibilidade de haver outro mecanismo adaptativo realizado por meio de modificações angulares do movimento e/ou por meio de outros grupos musculares. Uma possibilidade poderia ser as modificações do momento flexor do joelho ou as alterações de ativação de outros grupos musculares, como os adutores e rotadores laterais, que devem ser investigados em estudos futuros.

Sadeghi et al. relataram que os músculos do tornozelo e quadril são recrutados nas fases de apoio e balanço nos primeiros $50 \%$ do ciclo da marcha (15). Os autores sugeriram que a atividade extensora do quadril, que ocorre logo após o choque de calcanhar, está associada com o controle da aceleração do tronco (15). Essa condição pode ser desenvolvida e adaptada para a demanda diária normal, mas pode apresentar diferenças quando existe um aumento de perturbação, deixando o indivíduo suscetível a uma nova lesão. Apesar da população estudada não ter apresentado diferença entre membros acometido e não acometido e nem entre grupos, dentro da condição de teste realizada em uma velocidade de marcha confortável, novos estudos devem ser realizados para verificar o comportamento da ativação muscular em condições de alta demanda, assim como tempos de ativação diferenciados.

Um fenômeno apontado na literatura para explicar as mudanças nas estratégias posturais são os 
desequilíbrios musculares e a diminuição de força muscular $(4,22)$. Friel et al. afirmaram que a fraqueza da musculatura proximal pode estar associada às entorses recorrentes (5). Esses autores não encontraram diferença significativa entre a força dos extensores de quadril dos membros de indivíduos com e sem entorse unilateral de tornozelo, porém demonstraram haver fraqueza dos abdutores de quadril ipsilateralmente (5), o que reforça a hipótese de haver mecanismos adaptativos, com outros grupamentos musculares envolvidos. Como não foi objetivo deste estudo avaliar e comparar a força muscular, isso pode ter sido uma limitação de estudo.

Outro ponto a ser discutido refere-se aos resultados quanto à diferença de ativação entre membros direito e esquerdo, do grupo sem história de entorse do tornozelo. Jacobs et al. encontraram diferença de força entre membros dominante e não dominante, quanto à força de abdutores de quadril em indivíduos saudáveis, e defenderam que essa resultante não interferiu na funcionalidade e poderia ser interpretada como diferenças na dominância na utilização do membro (26). Dessa forma, estabelecer a dominância e mensurar a força muscular em estudos futuros pode ser relevante.

Do ponto de vista clínico, as informações obtidas neste estudo sugerem a necessidade em avaliar individualmente as estratégias biomecânicas diante da inter-relação segmentar entre o tornozelo e o quadril durante a marcha, em indivíduos que tenham sofrido entorse grau II do tornozelo. Uma vez que o pico de ativação muscular pode não ser o fator principal da necessidade de mudança da estratégia postural, a relação entre ativação muscular, força muscular, estratégias biomecânicas e propriocepção deve ser investigada.

\section{Conclusão}

Não foi observada na população avaliada diferença no pico de ativação do glúteo máximo durante a marcha em esteira, entre os grupos com e sem história de entorse de tornozelo. Esses achados sugerem que existem fatores adaptativos após o episódio de entorse do tornozelo para manter a marcha de forma funcional. Esses fatores podem estar relacionados com a dominância dos membros, alterações de força muscular e neuromusculares, que devem ser investigados em estudos futuros.

\section{Agradecimentos}

Ao professor Doutor Márcio Marçal por disponibilizar o aparelho dinamômetro Instrutherm DD-100, usado nas medidas de força da contração voluntária máxima isométrica.

\section{Referências}

1. You SH, Granata KP, Bunker LK. Effects of circunferential ankle pressure on ankle proprioception, stiffness, and postural stability: a preliminary investigation. J Orthop Sports Phys Ther. 2004;34(8):449-60. doi: $10.2519 /$ jospt.2004.1158

2. Hals TMV, Sitler MR, Mattacola CG. Effect of a semirigid ankle stabilizer on perfomance in persons with functional ankle instability. J Orthop Sports Phys Ther. 2000;30(9):552-9.

3. Leardini A, Benedetti MG, Catani F, Simoncini L, Giannini S. An anatomically based protocol for the description of foot segment kinematics during gait. Clin Biomech. 1999;14(8):528-36. doi:10.1016/S02680033(99)00008-X

4. Willems T, Witvrouw E, Verstuyft J, Vaes P, Clercq DD. Proprioception and muscle strength in subjects with a history of ankle sprains and chronic instability. J Athlet Train. 2002;37(4):487-93.

5. Friel K, McLean N, Myers C, Caceres M. Ipsilateral hip abductor weakness after inversion ankle sprain. J Athlet Train. 2006;41(1):74-8.

6. Ross SE, Guskiewicz KM, Yu B. Single-leg jump-landing stabilization times in subjects with functionally unstable ankles. J Athlet Train. 2005;40(4):298-304.

7. Berkowitz CPT, Kim DH. Fibular position in relation to lateral ankle instability. Foot \& Ankle Intern. 2004; 25(5):318-21.

8. McVey ED, Palmieri RM, Docherty CL, Zinder SM, Ingersoll CD. Arthrogenic muscle inhibition in the leg muscles of subjects exhibiting functional ankle instability. Foot \& Ankle Intern. 2005;26(12):1055-61.

9. Mangwani J, Hakmi MA, Smith TWD. Chronic lateral ankle instability: review of anatomy, biomechanics, pathology, diagnosis and treatment. The Foot. 2001; 11:76-84. doi:10.1054/foot.2001.0675 
10. Docherty CL, Gansnedert BM, Arnold BL, Hurwitz SR. Development and reliability of the ankle instability instrument. J Athlet Train. 2006;41(2):154-8.

11. Konradsen, L. Factors contributing to chronic ankle instability: kinesthesia and joint position sense. J Athlet Train. 2002;37(4):381-5.

12. Kaminski TW, Hartsell HD. Factors contributing to chronic ankle instability: a strength perspective. J Athlet Train. 2002;37(4):394-405.

13. Moussay S, Fransoo P. Relation entre la souplesse de la cheville et la flexibilité lombo-pelvieenne. Kinés. 2005;41:42-9.

14. Hubbard TJ, Kaminski TW, Griend RA, Kovaleski JE. Quantitative assessment of mechanical laxity in the functional unstable ankle. Med \& Sci Sports \& Exerc. 2004;36(5):760-6.

15. Sadeghi H, Sadeghi S, Prince F, Allard P, Labelle H, Vaughan CL. Functional roles of ankle and hip sagittal muscle moments in able-bodied gait. Clin Biomech. 2001;16(8):688-95.

16. Magee DJ. Avaliação músculo esquelética. 3a ed. São Paulo: Manole; 2002. p. 621-95.

17. Venturini C. Estudo de confiabilidade da força aplicada durante a mobilização anteroposterior do tálus e seu efeito sobre a goniometria de dorsoflexão do tornozelo. [dissertação]. Belo Horizonte: Universidade Federal de Minas Gerais; 2005.

18. Venturini C, Ituassu NT, Teixeira LM, Deus CVO. Confiabilidade intra e interexaminadores de dois métodos de medida de amplitude ativa de dorsiflexão do tornozelo em indivíduos saudáveis. Rev Bras Fisioter, 2006;10(4):407-11. doi: 10.1590/S141335552006000400008

19. Hermes HJ, Frekis B, Merletti R, Stegman D, Rau G, Klug CD, Hagg G. European recommendations for surface electromyography. Proceedings of the fourth general SENIAM workshop, 's Hertogenbosch. Italy: SENIAM; 1999.
20. Marchetti PH, Duarte M. Instrumentação em eletromiografia. Laboratório de Biofísica, Escola de Educação Física e Esporte. São Paulo: Universidade de São Paulo; 2006. [acesso em 29 mar. 2006]. Disponível em: http://demotu.org/pubs/EMG.pdf

21. Ocarino JM, Silva PLP, Vaz DV, Aquino CF, Brício RC, Fonseca ST. Eletromiografia: interpretação e aplicações nas ciências da reabilitação. Fisioter Brasil. 2005; 4(6):305-10.

22. Pacheco AM, Vaz MA, Pacheco I. Avaliação do tempo de resposta eletromiográfica em atletas de voleibol e não atletas que sofreram entorse de tornozelo. Rev Bras Med Esp. 2005;11(6):325-30.

23. Riemann BL, Myers JB, Lephart SM. Comparison of the ankle, knee, hip, and trunk corrective action shown during single-leg stance on firm, foam, and multiaxial surfaces. Arch Phys Med Rehabil. 2003;84(1):90-5. doi:10.1053/apmr.2003.50004

24. Ross SE, Guskiewicz KM. Examination of static and dynamic postural stability in individuals with functionally stable and unstable ankles. Clin J Sport Med. 2004;14(6):332-8.

25. Bullock-Saxton JE, Janda V, Bullock MI. The influence of ankle sprain injury on muscle activation during hip extension. Intern J Sports Med. 1994;15(6):330-4.

26. Jacob C, timothy L, Seeley M, Sterling W, Goodrich L. Strenght and fatigability of the Dominant and Nondominant Hip Abductors. J Athlet Train. 2005;40(3): 203-6.
Recebido: $18 / 01 / 2011$

Received: 01/18/2011

Aprovado: 13/05/2011

Approved: 05/13/2011 